\title{
Agricultural sustainability and food security
}

\author{
Naveen Kumar Arora ${ }^{1}$ \\ Published online: 12 November 2018 \\ (c) Society for Environmental Sustainability 2018
}

Agriculture is one of the most important practices developed by mankind for its survival and proliferation on earth. It was only due to agriculture that humans left the nomadic life style and settled in colonies now we know as cities, towns or villages. However, with unprecedented increase in population the pressure on agriculture also mounted. With limited space and fertile land available introduction of means such as chemical fertilizers and pesticides began in 19th and 20th centuries. This initially resulted in boom in the agriculture sector and we were able to feed major part of the ever growing human population. Despite a huge advancement in technology and un-precedent use of chemicals, recent surveys reveal that at present, around 870 million people are hungerstricken due to various resource scarcities and therefore FAO in 2012 reported that there is need to increase the agriculture production by $60 \%$ if we want to fulfil the needs of about 9 billion population in 2050 . Till date, to enhance the production of crops, farmers have mainly relied on use of chemical fertilizers and pesticides. Governments throughout the globe have played a major role in promoting the use of chemicals in the agriculture sector. It was and is believed that to feed the ever-increasing human population it is imperative to use chemicals in agriculture so as to enhance the yields and protect the crops against pests and pathogens. Moreover, introduction of transgenic crops through genetic modification were also implemented during the same period when chemicals were being injudiciously introduced in the fields i.e. during the era of green revolution (1950s and 1960s), which undoubtedly increased the production rate but at the cost of losing sustainability of environment in form of genetic erosion of indigenous varieties of crops and loss of fertile land. Excessive use of chemicals in agro-sector has also led to deleterious environmental problems like pollution of soil, air and water, soil salinity, development of pest resistance,

Naveen Kumar Arora

nkarora.bbau@gmail.com

1 Department of Environmental Science, BBA University, Lucknow, Uttar Pradesh, India loss of fertility of soil, and now is posing serious threats to food security, biodiversity and human health. Fertilizers and pesticides in particular have aggravated the extinction rates in the last 50 years causing elimination of keystones species specially affecting the microbial diversity and have escalated the rate of green house gases (GHG) causing global warming and climate change. To obviate these problems, it has become an indispensable need to administer such methods of farming which are ecologically compatible, holistic and are organic in nature. Sustainable approaches in agricultural sector are of utmost importance to improve the food security and nutrition problem around the globe.

Agricultural sustainability can be achieved by utilization and implementation of techniques of farming which would increase production of crops to meet demands of growing population while at the same time it would conserve and protect environment and its natural sources. While the food production at global level has been satisfactory in the last 50 years, recent approaches in context to agricultural development have not been impressive in achieving food security and in ensuring sustainability of environment. Therefore farming practices need to upgrade towards the use of biological agents in order to maintain the sustainability of agriculture and hence the environment. In this context, soil microbial diversity can be an important tool which acts as an indicator for soil health and plant's growth. Therefore, harnessing the role of beneficial soil microbes in perspective of agriculture can provide a measure towards achieving sustainability in the farming sector. Farming community since hundreds of years i.e. much before urea or dichlorodiphenyltrichloroethane (DDT) were discovered, knew that soil microbial diversity plays a very important role in maintaining the health and growth of crops. Crop rotation, mixing of fertile soils with non-fertile, and use of organic methods such as compost etc. have been in practice since time immemorial. Most of these sustainable techniques involve the importance and appreciation of beneficial soil microorganisms. But somewhere in between and because of huge pressure (of increasing population to feed) on the authorities/governments, these natural methods became extinct or 
rare in use. However, with the deterioration of agro-ecosystems as a result of use of agro-chemicals and other faulty practices we now know that beneficial soil microbes such as nitrogen fixers, phosphate solubilizers, metal chelators, phytohormones producers, and biocontrol agents have to be used for sustaining the agriculture productivity.

Agriculture has been an inevitable interaction between plants and useful microbes and as a result of the symbiotic nature of this relationship, plants are benefitted in a number of ways leading to enhanced growth and better yield. Among the huge bionetwork of soil microbiota, microbes (mainly bacteria and fungi) that thrive in the 'rhizosphere' i.e. the zone of soil around the vicinity of root system of plants, play as the cornerstone of agriculture and are known to defend plants from various abiotic (drought, flood and salinity) and biotic stresses (phytopathogens) in an eco-friendly manner. Hence, such microbes are known as plant growth promoting microorganisms (PGPM) and are one of the important tools when it comes to green solutions in agricultural sector. Production of growth regulators in form of secondary metabolites, nutrient solubilizers, antibiotics, ability to fix nitrogen and induction of induced systemic resistance (ISR) in plants are some of the important PGP traits known for these beneficial microbes. Use of PGPM in remediation of contaminated sites affected by pollutants like heavy metals, pesticides, polyaromatic hydrocarbons (PAHs), polycholorohydrocarbons (PCBs) and so on could be efficiently achieved and can be restored for further agricultural use. These rhizospheric microbes also reduce the effect of climate change by improving the productivity of barren or stress affected regions/soils. PGPM also balance nutrient cycling by increasing soil organic matter and reducing the chemical load in the soil. The ability to chelate micronutrients such as iron $(\mathrm{Fe}), \mathrm{Zinc}(\mathrm{Zn})$ and Copper $(\mathrm{Cu})$ by these microbes is another important aspect. PGPM are now being used to mitigate the problem of hidden hunger i.e. deficiency of nutrients particularly the micro-nutrients and vitamins, in the population. As per the data of FAO one out of nine persons on globe faces the problem of nutrient deficiency. The Sustainable Development Goals Report of 2018 by United Nations has reported that the proportion of undernourished people worldwide is increasing by the day. The ion chelating PGPM and nitrogen fixers are being used for biofortification of crops in a natural way. These multifarious characteristics make them possible substitutes to chemical fertilizers and pesticides and thus, they are now increasingly and successfully being used as bioinoculants around the world in sustainable manner.

First nitrogen fixing biofertilizer was based on symbiotic nitrogen fixing strain of rhizobia and was patented in the year 1896 under the brand name "Nitragin" for 17 different leguminous plants. Presently, over 2000 tons of rhizobia based inoculants are being produced every year equivalent to USD 50 million. Nitrogen based biofertilizers hold the largest market share of $78.7 \%$ (globally amongst the total biofertilizer market) and is expected to rise with an annual growth rate of $13.25 \%$ by 2020 . As per the recent reports, global biofertilizer market was estimated as USD 787.8 million in 2016 whereas biopesticide market was USD 3.3 billion in 2017. Fascinatingly, nature has provided countless avenues in this area as only $1-2 \%$ of cultured microbes have been explored till date and there is still immense range of diversity which needs to be studied and explored. Further studies of plant microbiome, and integrating it with 'omics' techniques such as transcriptomics, metagenomics and metabolomics could improve our knowledge in unravelling the genetic diversity, population structure and dynamics of these beneficial microbes in order to exploit them in more diverse ways as bioinoculants. Also, enhanced research in formulating these bio-based products and their delivery systems are other key areas of research. Use of improved microbial inoculants (both biopesticides and biofertilizers) can be the excellent tools of organic farming to curb the negative impacts of their chemical counterparts. Microbe based inoculants are lower in cost, protect indigenous flora and fauna and other natural resources, have less or no harmful by-products, improve soil organic matter and above all, maintain sustainability of ecosystems. Despite the huge benefits of bioinoculants/biofertilizers/biostimulants/biopesticides, whatever we name them, these biological formulations are still only a miniscule part of the global agro-market and chemicals still rule throughout the globe. Hence there is an urgent need that organizations, governments, authorities, scientists, agriculturists and the farming communities come together and help in formulating policies to increase the use of these sustainable products in agro-ecosystems in multifarious ways to enhance the crop productivity, combat the impact of climate change, increase the productivity of wastelands and fight biotic and abiotic stresses in an ecofriendly manner. Promotion of harmful chemicals has to be brought down and farmers must be educated (particularly in developing countries) for the use of biological alternatives. It is also important to regulate the quality of bioinoculants so that the end-users get confidence in replacing the harmful chemicals with the biological methods. Again all the stake holders need to take part to formulate and implement strict policies so as to promote the use of quality products.

The Sustainable Development Goals Report (2018) by United Nations states that in order to achieve sustainability, upliftment of economic growth is necessary and is directly linked to achieve food security, improve nutrition and sustainable agriculture. To achieve the goals of food security and sustainability in agricultural sector, emphasis has to be made on the use of traditional biological methods amalgamating them with the recent innovations in biotechnology and bio-engineering, so as to utilize the un-culturable microbes, 
their genes and metabolites as well as improving the industrial production of bioinoculants, respectively. At present we know that biological alternatives are the only ways to save us from disaster in agriculture and lead to food security via sustainability. Sustainable agriculture techniques involving latest biotechnological tools and classic methods are the key focus areas of the journal "Environmental Sustainability". The journal is committed to promote the research which focuses on sustainability of agro-ecosystems and help in attaining food security. 